\title{
Protée
}

\section{Construction dans la filiation}

W ou le souvenir d'enfance

\section{Anne Élaine Cliche}

Volume 33, numéro 3, hiver 2005

Filiations

URI : https://id.erudit.org/iderudit/012505ar

DOI : https://doi.org/10.7202/012505ar

Aller au sommaire du numéro

\section{Éditeur(s)}

Département des arts et lettres - Université du Québec à Chicoutimi

ISSN

0300-3523 (imprimé)

1708-2307 (numérique)

Découvrir la revue

Citer cet article

Cliche, A. É. (2005). Construction dans la filiation : $W$ ou le souvenir d'enfance. Protée, 33(3), 87-107. https://doi.org/10.7202/012505ar

\section{Résumé de l'article}

La réécriture du souvenir d'enfance que constitue la description de $\mathrm{W}$, île gouvernée par l'idéal olympique, est présentée comme une construction qui permet de restaurer une filiation rompue, abolie, oubliée. Georges Perec y met en acte une violence qui attente au corps maternel perdu. d'utilisation que vous pouvez consulter en ligne.

https://apropos.erudit.org/fr/usagers/politique-dutilisation/ 


\title{
CON STRU CTION DAN S LA FILIATIO N W OU LE SOUVENIR D'ENFANCE
}

\author{
ANNE ÉLAINE CLICHE \\ J'ai tant souffert d'être "le fils», que ma première ceuvre ne peut être \\ que la destruction totale de tout ce qui m'engendra (le bourreau, \\ thème connu, automaïentique).
}

Georges Perec à Jacques Lederer, le 7 juin 19581

«LE LIVRE DE LA DÉFILIALITÉ»²

On a tellement étudié, commenté et analysé l'autobiographie de Georges Perec, qu'il parait sans doute bien ambitieux d'espérer ajouter à cette somme quelque contribution que ce soit. Et pourtant... La lecture de W ou le souvenir d'enfance 3 , malgré les lumineux éclairages produits par les travaux sur les avant-textes ${ }^{4}$, sur Perec et la psychanalyse ${ }^{5}$, sur Perec et la judéité ${ }^{6}$, sur le traitement de la mémoire, de l'histoire, sur l'invention de l'identité ou l'écriture concentrationnaire ${ }^{7}$; malgré les commentaires nombreux qui ont établi les rapports d'analogie et de divergence entre la description de l'île W et les camps nazis, et qui ont reconnu dans la fiction une manière singulière - métonymique? allégorique? - de raconter l'anéantissement de la mère; malgré, donc, cette abondance d'articles, de monographies, de collectifs, la lecture de ce livre demeure toujours énigmatique.

Que reste-t-il à dire? Je ne sais trop. Mais ce qu'il y a d'inquiétant - d'intéressant et d'étonnant - avec W ou le souvenir d'enfance, c'est que nous gardons l'impression, quand ce n'est pas la certitude, que ce livre est en reste de toute lecture, qu'il nous a échappé, que la perte qui s'y inscrit est devenue celle-là même que nous ressentons devant lui. Perte, déperdition, désertion, dérobement qui, certes, ressemble à un jeu, comme toujours chez Perec, mais s'éprouve aussi comme une agression, une entreprise de destruction, alors que tout, dans ces pages, se donne pour restauration, fantasme, réparation. S'il s'agit d'un témoignage, son statut est pour le moins problématique, puisque le «témoin» est un enfant juif, caché puis baptisé pendant la guerre, seul survivant de sa lignée, le père étant «mort pour la France», au début des hostilités, et la mère déportée, disparue dans les cendres d'Auschwitz. 
De tous ces événements, le «témoin » n'a rien vu, et n'a découvert la vérité, d'ailleurs insaisissable, qu'à la fin de l'Occupation ${ }^{8}$.

Je reprends donc à mon tour cette lecture, pour m'y perdre sans doute, mais peut-être avant tout pour entendre quelque chose de la filiation qui accède rarement à ce mode radical d'exposition et d'exhibition que pratique Perec; mode selon lequel la représentation est détournée et pourtant frontale, manifestement faussée, masquée, et cependant offerte dans la violence trouble d'un registre où la pudeur ne contredit en rien l'obscène. Il s'agira donc de relire cette écriture volontairement cryptique, voire encryptée, qui se dispose d'ailleurs elle-même, avec ruse et candeur, comme un travail d'interprétation à saisir dans sa puissance d'acte, de fiction, de construction, pour parler comme Freud qui désignait ainsi l'interprétation psychanalytique au moment où elle propose au sujet un fragment d'histoire inventé voire faux - tenant lieu de souvenir de quelque événement dont «rien» ne subsiste, et qui en prend la place ${ }^{9}$.

Dans W ou le souvenir d'enfance, Perec noue deux narrations. L'une en italique qui met en scène l'histoire de Gaspard Winckler et le fantasme de l'île W, l'autre en caractères romains prise en charge par Georges Perec qui raconte l'enfance, la mort du père, la disparition de la mère, quelques souvenirs de la rue Vilin, puis la vie en zone libre, en pension à Villardde-Lans, alors qu'il est recueilli par la famille de la sour du père. Ce sont ces deux «trames", l'autobiographie d'enfance fragmentée en petits chapitres et la fiction $\mathrm{W}$ rédigée elle aussi en brefs chapitres reconnaissables à leurs caractères italiques, que Perec tisse en alternance. Cette écriture s'organise dès lors selon un processus qui met en résonance des mouvements manifestement contraires: d'une part, celui d'une fiction linéaire soutenue, dans laquelle Gaspard Winckler se voit confier la mission de retrouver un enfant sourd-muet porté disparu à l'occasion d'un naufrage près de la Terre de Feu, enfant dont il aurait reçu l'identité après sa désertion, lors de son refuge en Allemagne; d'autre part, celui d'une dissémination et d'une recollection de bribes plus ou moins précises de souvenirs reformulés et commentés jusqu'à l'annulation.

La mission de Gaspard Winckler trouvera une conclusion imprévue après une brusque interruption qui clôt la première partie du livre et laisse en blanc le fragment autobiographique que l'on attendait. Cette conclusion compose la seconde partie de la fiction en organisant, chapitre par chapitre, la description minutieuse de la société W, île présumée du naufrage. Malgré sa fidélité à la linéarité narrative qui le rattache de toute évidence à l'histoire de Gaspard Winckler (le vrai et le faux), le récit de la vie à W apparaît pourtant sans commune mesure avec ce qui le précédait. Les deux trames alternées du début, qui aboutissent brusquement, au milieu du livre, dans un trou représenté par trois points de suspension entre parenthèses et placés au centre d'une page blanche, reprennent en effet, après ce blanc, leur jeu d'alternance, mais sur un autre registre. En quatrième de couverture, Perec a pris soin d'indiquer que c'est

[...] dans cette rupture, cette cassure qui suspend le récit autour

d'on ne sait quelle attente, [que] se trouve le lieu initial d'où est sorti ce livre, [à] ces points de suspension [...] se sont accrochés les fils rompus de l'enfance et la trame de l'écriture.

Mais le livre se divise encore sur un autre plan qui correspond à trois temps de l'écriture: deux courts textes apparaissent, en effet, en caractères gras dans la première partie autobiographique et se présentent comme «datant de plus de quinze ans» (Wse: 45-46). Ils sont commentés par vingt-six notes contemporaines de la rédaction des fragments autobiographiques (1971-1974). On peut donc supposer que ces textes remontent aux années 1956 1957. Ils constituent une tentative de rassembler les informations que le sujet détient respectivement sur son père et sur sa mère; informations fragilement compilées à partir de photos et de commentaires approximatifs. L'histoire de Gaspard et de W remonte, quant à elle, à la publication en feuilleton que Perec a livrée à la revue de Maurice Nadeau, La Quinzaine littéraire, entre septembre 1969 et août 1970. 
Pour sa part, le narrateur Perec, travaillant à partir de documents divers et fragmentaires, se présente comme frappé d'amnésie, dépositaire de souvenirs morcelés, désarrimés et pour la plupart incertains. Il joue manifestement l'association libre, la remémoration en acte. L'assertion quasi triomphale, qui ouvre cette trame autobiographique et qui ressemble autant à une plainte qu'à un défi - «Je n'ai pas de souvenir d'enfance» (Wse: 17) -, est présentée comme la phrase-écran derrière laquelle il se serait longtemps protégé, caché, voire disculpé d'une histoire d'autant plus dure à reconnaitre qu'elle serait douloureuse ou impossible à reconstituer. Cette phrase installe ostensiblement l'écriture de ce livre dans le registre du «rien" à recouvrer comme à recouvrir, elle tient lieu de causalité à ce qui se donne ici pour depuis toujours empêché et soumis au travail incessant de (re)construction.

Je n'ai pas de souvenir d'enfance. Jusqu'à ma douzième année à peu près, mon histoire tient en quelques lignes: j'ai perdu mon père à quatre ans, ma mère à six; j'ai passé la guerre dans diverses pensions de Villard-de-Lans. En 1945, la sœur de mon père et son mari m'adoptèrent. Cette absence d'histoire m'a longtemps rassuré: sa sécheresse objective, son évidence apparente, son innocence, me protégeaient, mais de quoi me protégeaient-elles, sinon précisément de mon histoire [...] qui, on peut le supposer, n'était ni sèche, ni objective, ni apparemment évidente, ni évidemment innocente? [...] L'on n'avait pas à m'interroger sur cette question [...] j'en étais dispensé: une autre histoire, la Grande, l'Histoire avec sa grande hache, avait déjà répondu à ma place: la guerre, les camps. (Wse: 17)

Cette autobiographie ne constitue pas cependant le point de départ du livre; elle survient dans l'écho d'une première écriture, toute fantasmatique et fictive, qui ouvre le livre sur un ton singulier, marqué par le doute, dont le travail de mémoire serait en quelque sorte la conséquence, le produit ou, plus précisément encore, la solution.

Tout ce travail autobiographique, dira en effet Perec, s'est organisé autour d'un souvenir unique qui, pour moi, était profondément occulté, profondément enfoui et d'une certaine manière nié. 10
On apprendra que c'est le souvenir d'un fantasme oublié, puis retrouvé un soir à Venise, qui est repris dans la seconde partie de cette histoire d'enfant perdu-recherché. Le narrateur, Gaspard Winckler, raconte pour commencer comment, ayant déserté et obtenu une fausse identité, il reçoit, de la part d'un personnage obscur nommé Otto Apfelstahl, d'abord une lettre au blason énigmatique qui le convoque à l'hôtel Berghof, puis la mission de retrouver le corps disparu de l'enfant dont il porte le nom. La rencontre avec Apfelstahl constitue toute le première partie de ce feuilleton qui donne son cadre à la seconde, entièrement consacrée à la description de l'île W située au large de la Terre de Feu et gouvernée par l'idéal olympique.

À treize ans, j'inventai, racontai et dessinai une histoire. Plus tard, je l'oubliai. Il y sept ans, un soir, à Venise, je me souvins tout à coup que cette histoire s'appelait «W» et qu'elle était, d'une certaine façon, sinon l'histoire, du moins une histoire de mon enfance. En dehors du titre brusquement reconstitué, je n'avais pratiquement aucun souvenir de W. Tout ce que j'en savais [...]: la vie d'une société exclusivement préoccupée de sport, sur un îlot de la Terre de Feu. [...] Je retrouvai plus tard quelquesuns des dessins que j'avais faits à treize ans. Grâce à eux, je réinventai $W$ et l'écrivis, le publiant au fur et à mesure en feuilleton [...]. W ne ressemble pas plus à mon fantasme olympique que ce fantasme ressemble à mon enfance. Mais dans le réseau qu'ils tissent comme dans la lecture que j'en fais, je sais que se trouve inscrit et décrit le chemin que j'ai parcouru, le cheminement de mon histoire et l'histoire de mon cheminement.

Il faudra dire ici, bien sûr, ce que permet de penser cette réécriture d'un fantasme infantile oublié. Ce qu'elle suppose aussi de restauration, de rédemption et de négation. Mais ce que le livre nous apprend dès l'ouverture, c'est le «cheminement» qui a conduit du fantasme au feuilleton, puis à la composition d'une structure qui reprend intégralement le texte du feuilleton pour y intercaler, suivant une discrète technique de concordances, des fragments autobiographiques qui trouvent leurs points de «suture» dans la matrice fictionnelle ${ }^{11}$. Dans la trame 
autobiographique, la scène de la séparation à la gare de Lyon, entre l'enfant - envoyé par un convoi de la Croix-Rouge en zone libre - et sa mère, apparaît comme un point de butée de la mémoire incapable de retrouver le souvenir de la mère, de son corps, de son visage, de son attachement à l'enfant ${ }^{12}$. Tous les systèmes d'attache et de suspension évoqués dans le livre semblent d'ailleurs se substituer au lien, impossible à reconstituer, que serait la main de la mère tenant celle de l'enfant.

Conséquemment, les filins, suspentes et autres systèmes d'accrochage qui occupent les souvenirs, et dont la description détaillée du fonctionnement semble procurer un intense plaisir, constituent une véritable métaphore; on ne s'étonnera pas de les retrouver convertis en instruments de torture dans le fantasme W. Si la filiation se définit minimalement comme ce qui nous rattache aux ascendants et aux descendants et nous inscrit dans un continuum généalogique, les «fils rompus», qui sont au principe de la parole de Perec, provoquent une déclinaison de la filialité sur le mode de la «filature» et du roman policier. Ce qui est recherché est aussi bien la mémoire et l'origine que l'enfant abandonné, ou mort ou survivant, qu'est ce Gaspard Winckler ayant donné son nom au déserteur anonyme qui dit «Je» avant de s'abîmer à son tour dans la voix off qui préside à la description «ethnologique» de l'île W. C'est ce «lieu» $\mathrm{W}$, magistralement exhumé dans ce livre, et pourtant obstinément irrepérable, que je souhaite traverser comme un désert: celui de la filiation, autant dire celui déserté par la filiation et devenu zone de travail de la défilialité. Il s'agira de voir comment le fantasme infantile passe à l'écriture, et donc à un second degré de symbolisation, en maintenant à vif la jouissance en cause. Il s'agira de voir comment, dès lors, quelque chose de "l'enfant survivant» du rêve, pour reprendre l'expression de Freud qui trouve ici une portée doublement signifiante, demeure - à notre «surprise» - actif dans la jouissance de l'adulte écrivain, et ce, malgré les nombreux énoncés du narrateur autobiographe, qui prétendent corriger, ajuster, rectifier la vision d'enfance ${ }^{13}$.

\section{L'ENFANT-PÈRE}

La découverte de la tombe de mon père, des mots PEREC ICEK JUDKO suivis d'un numéro matricule, inscrits au pochoir sur la croix de bois, encore tout à fait lisibles, m'a causé une sensation difficile à décrire: [...] l'étonnement de voir mon nom sur une tombe (car l'une des particularités de mon nom a longtemps été d'être unique: dans ma famille personne d'autre ne s'appelait Perec) [...].

(Wse: 58)

La reprise fictionnelle du fantasme infantile, recadré par l'histoire de Gaspard Winckler partant à la recherche de "celui qui lui a donné son nom", est en soi une épreuve, autant pour celui qui écrit - les avants-textes gardent la trace de cette souffrance aiguë attachée à l'écriture de $\mathrm{W}^{14}$-, que pour quiconque s'aventure dans cette lecture, innocemment livré au discours qui se construit comme un leurre et bientôt comme un piège. La voix narrative désormais masquée - est-ce celle de l'enfant retrouvé vivant sur l'île, le vrai Gaspard Winckler? ou celle du faussaire? ou celle de l'enfant mort ET survivant qui ne peut parler qu'à partir de cette place excentrée? -, cette voix, donc, apparemment impersonnelle et imperturbable, entame la description de la société W entièrement soumise aux lois du sport. La froideur scientifique du narrateur, obsessionnellement occupé à transmettre les calculs et les chiffres sur lesquels se règlent les corps soumis aux impératifs de la compétition, se révèle peu à peu dans sa complaisance maniaque qui apparaît bientôt entretenir une complicité de structure avec le système abject qu'il décrit. Le lecteur est d'ailleurs lui-même conduit insidieusement à entrer dans les rouages d'une jouissance à laquelle il s'aperçoit trop tard avoir goûté. Une sorte de jubilation retenue, d'admiration réservée, imprègne en effet la présentation prétendument objective des règles qui sévissent à $\mathrm{W}$; présentation qui étale au grand jour les «symptômes" perequiens par excellence: classer, dénombrer, répertorier, combiner, agencer; véritable étalage des contraintes qui, chez Perec, sont fonction d'engendrement et de création, et qui trouvent dans ce fantasme recomposé une 
application corporelle directe. Leurre, piège et cruauté seraient bien les modalités du roman familial perequien.

Tout se passe comme si la contrainte oulipienne trouvait enfin, dans cette fiction d'olympiades, sa matière première qui est corps et, ce faisant, corps de la lettre. Lisant Perec, il faut toujours en passer par une interprétation de la lettre - pour ne pas dire par une lecture à la lettre - à laquelle nulle herméneutique ne saurait correspondre. Et c'est encore la lettre, la dimension littérale du fantasmefiction, qui engendre, dans $W$ ou le souvenir d'enfance, l'autobiographie, l'histoire ou sa mémoire, et non l'inverse $^{15}$. La filiation perequienne n'est donc pas tant généalogique que littérale (et littéraire), comme on le verra. L'effacement quasi radical des parents, des ancêtres, non seulement de la mémoire de l'écrivain, mais aussi de sa quête de l'origine, montre bien qu'à la question «D'où suis-je venu?», ne répond pas tant une lignée, une transmission, un héritage, qu'une fiction qui trouve sa texture aussi bien dans la construction d'un fantasme entièrement mû par le «W» que dans la corporéité tyrannique de la lettre: qu'elle apparaisse toute seule comme cette fausse lettre hébraïque qui se donne pour le premier souvenir (Wse: 27) ${ }^{16}$; qu'elle soit frappée d'interdit, comme le «E» dans La Disparition, ou, au contraire, devenue obligatoire, comme le «E» dans Les Revenentes; qu'elle soit surtout l'enjeu de contraintes et de torsions, de calculs ou de métamorphoses, au point de faire de l'écriture le terrain de violences et de massacres incessants. La filiation perequienne est jonchée de cadavres - certes, comme toute filiation -, mais ces cadavres sont constamment soumis à la violence symbolique que s'impose l'écrivain dans son travail de résurrection-assassinat, selon lequel c'est le symbolique lui-même qui est visé, sa puissance combinatoire quasi infinie et révélée par la lettre, sa fonction opératoire inconsciente.

Cette violence meurtrière - par ailleurs si manifeste dans La Vie mode d'emploi et dans La Disparition - passe en effet par le maniement obsédé de la lettre, support de l'homophonie, mais aussi enjeu d'un retranchement du sens et du langage. La lettre, on le sait, n'est pas déchiffrée en tant que telle dans l'acte de lecture qui, justement, nécessite ce refoulement du littéral pour produire l'assomption du sens. La fascination de Perec pour les mots croisés, son engagement dans la fabrication des grilles (fabrication qui précède la formulation des définitions), qu'il éprouve comme un «système de contrainte primaire où la lettre est omniprésente mais d'où le langage est absent» ${ }^{17}$, apparaît comme une entreprise de vidage du sens, effet d'un désir d'emprise sur le symbolique. On peut certainement dire, de là, que toute l'écriture de Perec est formellement hantée par la filiation, par le désir d'en découdre avec la filiation pour ne pas dire d'en rendre le décousu, d'en jouer la cassure, la brisure, pour enfin pouvoir la dire et s'en faire le signataire:

Dans le caveau des miens, plongeant mes pas nocturnes/J'ai compté mes aieux suivant leur vieille loi/ [...] À peine une étincelle a relui dans leur cendre/C'est en vain que d'eux tout le sang me fait descendre;/Si j'écris leur mémoire, ils descendront de moi. 18

Les conditions de la mémoire sont posées, dans $W$ ou le souvenir d'enfance, à partir de diverses stratégies qui vont de la description minutieuse de photographies et, plus généralement, de l'usage répété d'un matériel fragmentaire et de documents «officiels» dont il s'agit de rendre compte, sur le mode le plus objectif possible, à l'écriture fictionnelle décrivant un système totalitaire exempt de toute référence manifeste ou explicite à l'histoire personnelle de celui qui raconte. Ces deux «méthodes» semblent vouées à l'échec, la mémoire refusant toute restauration et ne libérant jamais que ces «points d'encrage», c'est-à-dire ces «lieux» par où elle s'est abîmée ${ }^{19}$. Or, ces lieux sont des lettres: W, X, $\mathrm{E}, \mathrm{H}$, etc., composant tout un alphabet secret et fictif qui ne cesse d'être mis à l'épreuve depuis que l'écriture occupe, dans la vie de Perec, la place du commencement:

J'éris parce qu'ils ont laissé en moi leur marque indélébile et que la trace en est l'écriture: le souvenir est mort à l'écriture; 
l'écriture est le souvenir de leur mort et l'affirmation de ma vie.

(Wse: 63-64)

Cette écriture qui tient lieu de filiation, on l'a certes reconnue dans ses effets nombreux d'engendrement, où l'intertextualité prend, pourrait-on dire, fonction de Père et fait Loi sur tout le texte perequien ${ }^{20}$. Je voudrais ici faire entendre la part obscure qui fabrique du Père avec l'«enfant survivant» qu'on retrouve, comme le soulignait Freud, avec surprise dans le rêve et qui, chez Perec, fait retour dans l'écriture fictionnelle.

La «surprise» (Überraschung) dont Freud fait état atteste suffisamment le fait que cette survie n'aille pas de soi [...].

Pourquoi une telle surprise? À un premier niveau de lecture on peut attribuer cette réaction au fait que, selon l'opinion courante, le devenir-adulte est représenté comme abandon ou destruction de l'enfance. D'où l'étonnement devant la survie d'une enfance qu'on aurait pu croire enterrée. Mais l'expression weiterlebend (encore vivant) peut également renvoyer, si on la situe dans le paysage imaginaire déployé dans L'Interprétation des rêves, à toutes ces menaces infanticides planant sur l'enfant. [...] Il y a certes une différence décisive entre le weiterleben (viure encore, continuer à viure) et le überleben (survivre), mais, dans l'une comme dans l'autre expression, la continuation de la vie est rencontrée comme surprenante, comme faisant saillir les menaces mortelles qui surplombent cette vie sursitaire. $^{21}$

Il est certain que l'infanticide est au noyau du fantasme infantile qui produit l'écriture de $\mathrm{W}^{22}$. Et cet infanticide comme menace et désir, mais aussi vœu accompli, réalisé dans l'histoire du sujet, détermine le registre de cette fiction. C'est la mise à découvert de ce qui, dans ce texte, est à la fois voilé et dévoilé, qui permettra d'approcher la violence, la cruauté, la vengeance et la haine qui opèrent dans la filiation. Les motions et impulsions qui se trouvent, par cette écriture spécifique, exposées en négatif à défaut d'être formulées, on pourrait croire que, chez Perec, elles tiennent lieu d'histoire autant qu'elles donnent lieu à des histoires... décalées, «à côté» de leur surgissement. C'est ce singulier destin, cette manière qu'a l'écrivain d'en faire sa chose "absente», sa matière fuyante, qui finit par les «rendre», les inventer, assurément méconnaissables, re-créées qu'elles sont par le jeu, les contraintes, les miroirs, les cadrages.

Perec envisageait le travail de défilialité pour son premier roman, autant dire au principe même du passage à l'écriture, du devenir-écrivain, comme "l'achèvement définitif des spectres du passé» ${ }^{23}$. Mais de quoi s'agit-il au juste? D'automaïeutique, ou encore d'auto-engendrement, thème connu en effet.

Toutefois, cette pratique apparaît ici inséparable de la torture - souffrance et blessures à soutenir -, dont le dispensateur et bourreau est cet «inengendré fils de personne» (Wse: 25) désirant encore et toujours se faire maître de sa naissance et de sa destinée. On peut déjà entrevoir le programme sadomasochique que ce travail suppose.

Car imaginer $W$, c'est fatalement en devenir d'une certaine manière responsable: on fait travailler en soi, même si c'est dans la douleur, le bourreau virtuel; on ressuscite le lien abject qui lie la victime au tortionnaire. 24

On doit relire W, l'histoire de l'île et de ses olympiades, comme l'invention d'un lieu pour accomplir cette destruction... inachevable, qui ne trouve d'ailleurs pas d'autre achèvement que l'interruption au bout d'un crescendo impitoyable.

FORT/DA

L'enfant avait une bobine en bois avec une ficelle attachée autour. [...] il jetait avec une grande adresse la bobine, que retenait la ficelle, par-dessus le rebord de son petit lit [...] où elle disparaissait, tandis qu'il prononçai le son o-oo-o riche de sens ["parti»(fort)]; il ramenait ensuite la bobine [...] en tirant la ficelle et saluait alors sa réapparition par un joyeux «voilà» (Da). 25

Il y a, dans $W$ ou le souvenir d'enfance, un mouvement, une oscillation qui s'apparente au fort/da de l'enfant freudien - c'est-à-dire à un jeu de jeter/ reprendre, faire disparaitre/retrouver, lâcher/rattraper -, actualisé par une écriture alternée et différentielle. D'une part, un récit d'aventures linéaire qui tourne au 
catalogue de lois perverses; d'autre part, un récit de souvenirs arrachés au néant, annulés par le doute ou encore falsifiés. Quelle part représente le fort (loin), et quelle part le da (voilà)? Dans la dialectique du jeu qui met en mouvement la bobine (la mère) perdue puis retrouvée, il semble que tout s'organise pour faire du fantasme-fiction $\mathrm{W}$ le lieu de retrouvailles, puisque Gaspard Winckler, le faux, l'adulte, est envoyé en mission pour trouver Gaspard Winckler, le vrai, l'enfant, et que l'histoire de l'île W arrive bel et bien en lieu et place du récit de cette potentielle (re)trouvaille. Mais de quelles retrouvailles s'agit-il, au juste? Le récit autobiographique qui a les caractéristiques du lointain, de l'oublié, de l'effacé, du disparu, et qui s'écrit manifestement dans le style du "souvenir-écran», ne nomme pas tant la perte qu'il la joue, l'actualise. Oui, ce sont bien les parents qui sont perdus, morts. Oui, ce sont la mère et le père qui manquent cruellement à cet enfant nommé Georges Perec. Et oui, c'est bien cet enfant "sourd-muet», aboli dans le naufrage de l'Histoire, qui reste en souffrance du texte et des mots pour souffrir. Est-ce à dire pour autant que ce sont eux qui sont retrouvés et même qu'il faudrait retrouver? Et qu'est donc ce «Eux» que Perec a vocalisé en «E» pour à la fois en liquider la trace, en abolir la présence, et ressusciter l'impératif de leur mémoire par la puissance d'un interdit ( $\mathrm{La}$ Disparition)? C'est à cette lettre encore qu'est dédié $W$ ou le souvenir d'enfance, («Pour $\mathrm{E} »)$, comme s'il s'agissait en effet d'écrire pour rendre compte de ce qu'ils furent et dont il ne reste rien... que le rien. "Je ne sais pas si je n'ai rien à dire, je sais que je ne dis rien; [...] je sais que ce que je dis est blanc, est neutre, est signe une fois pour toutes d'un anéantissement une fois pour toutes» (Wse: 63).

Chez Perec, retrouver ce n'est jamais que trouver une piste nouvelle, la forme inédite et inespérée d'une énigme insoluble et insistante, quand ce n'est pas tout simplement trouver la mort, en l'espèce du massacre, du meurtre, du naufrage ou de la disparition. Ce statut du da (voilà) est en quelque sorte bien particulier, puisque ce qu'il s'agit de retrouver, ce sont bien sûr les traces d'une disparition, les contours et les bords d'un évanouissement, d'un rien qu'il faut bien reconnaître pourtant comme sien, non pas tant comme nom - car le nom se décline, se porte, se ramifie, s'étymologise, s'échange - que comme corps ou comme voix (corps de l'enfant aboli dans le temps, voix qui pouvait porter ce corps, l'envelopper, l'abriter). C'est bien cette voix que Perec a cherchée, a re-trouvée; une voix qui devait bien lui venir de quelque part et qu'il a déposée dans le corps de la mère fictive, la cantatrice Caecilia Winckler, qui accompagne l'enfant au moment du naufrage, cantatrice qui est aussi à l'origine de cette histoire W, puisque c'est elle qui aurait fait transmettre les papiers de son enfant au déserteur réfugié. Cette voix perdue semble en tout cas se retrouver par l'écriture, une écriture que le «voilà" perequien désigne à merveille:

[...] il rabâchait sans fin un mot idiot qu'il n'arrivait jamais à

saisirr: voilà, ou vois-là, ou voyou, ou voyal? qui, par associations

provoquait un amas, un magma incongru: substantifs, locutions, slogans, dictons, tout un discours confus, brouillon, dont il

croyait à tout instant sortir, mais qui insistait, imposant

l'agaçant tourbillon d'un fil vingt fois rompu, vingt fois cousu, mots sans filiation, où tout lui manquait, la prononciation, la transcription, la signification, mais tissant toujours un flux, un flot continu, compact, clair: impact sûr, intuition, savoir s'incarnant soudain dans un frisson vacillant, dans un flot qu'habitait tout à coup un signal plus sûr, mais qui n'apparaissait qu'un instant pour aussitôt s'abolir. 26

Je veux entendre ce passage de La Disparition comme celui qui décrit le plus admirablement l'écriture de Perec, à la fois engluée dans l'interdit de la mémoire, égarée dans les jeux de mots incongrus, les locutions, les lipogrammes et anagrammes, et tissant avec art, assurance et brio son flot de parole limpide, juste et insaisissable. "Mots sans filiation», écrit Perec, mots sans autre filiation que la contrainte qui les engendre et qui est la part la plus rusée, la plus habile de la torture toujours liée au travail d'abolition, de destruction totale évoqué plus haut, et qui n'est pas sans susciter, on le comprend maintenant, un retour de l'aboli, surgi comme "un signal plus sûr», et déjà inaudible. On comprend que c'est précisément cette 
maîtrise qui permet à l'enfant du jeu, comme le disait Freud, de se dédommager pour le renoncement pulsionnel, que lui impose le départ de sa mère, en inversant sa passivité devant les événements en un rôle actif.

Mais l'on peut encore proposer une autre interprétation. En rejetant l'objet pour qu'il soit parti, l'enfant pourrait satisfaire une impulsion, réprimée dans sa vie quotidienne, à se venger de sa mère qui était partie loin de lui. ${ }^{27}$

On verra ici comment le livre de Perec actualise, de manière remarquable, cette vengeance devenue «jeu». Il ne faut d'ailleurs jamais manquer de rappeler que, dans ce lancer/tirer de la bobine, ce sont les vocables (o-0-o-0/a-a-a-a) qui inscrivent la marque différentielle qui procure le "gain de plaisir"; ce sont bien les signifiants, et donc ce qui «encre» l'enfant dans la Loi symbolique (le Père), qui tracent l'incise, les bords du trou par où la mère peut disparaître.

Le livre de la défilialité est donc aussi, on s'en doutait, celui des filiations, c'est-à-dire une pratique qui cherche à refaire, à maîtriser mais aussi à venger l'origine; une pratique qui fabrique ses structures de la parenté, ses principes de génération. On pourrait certainement parler d'CEdipe, mais cela ne dirait rien des modalités particulières en jeu ici. «Tuer le père pour accéder à la mère» est un programme qui semble en effet trouver, chez Perec, une formulation d'autant plus inquiétante qu'elle est apparemment indépassable: «Anéantir la victime pour rejoindre la jouissance du bourreau». Programme selon lequel l'Eedipe n'en finira plus de reconfigurer ses impasses. "Quand je pense que cette conasse de langue française désigne de la même manière Pair et Père - Bouah [...]. La Nuit est l'achèvement définitif des spectres du passé» 28 .

À Jacques Lederer qui lui envoie l'anagramme de son nom ( "Georges Perec = Égorge ce père» $\left.{ }^{29}\right)$, Perec répond: «je m'y emploie». Toute l'œuvre de Perec peut être lue selon cette logique de l'attentat contre le spectre du père tué, attentat contre un mort qui vous confine "officiellement» à la place de fils. Le jour de ses 22 ans (le 7 mars 1958), alors qu'il fait son service militaire près de Pau dans les Basses-Pyrénées, Perec écrit à Jacques Lederer: «Soir. Tir de nuit. Étrange et féérique ballet de lumières falotes des silhouettes à peine sorties de la nuit. Des images se superposent. Égorge ce père! une balle dans le ventre...» ${ }^{30}$. C'est bien lui, Georges Perec, qui, dans le fantasme, est visé, dans son nom devenu impératif de parricide. Mais c'est aussi ce père qui, là, fait retour; ce père mort au combat d'un éclat d'obus, dont l'enfant a rêvé «les morts" glorieuses, et dont l'adolescent a voulu effacer toute gloire (Wse: 48). On ne sait plus si la balle dans le ventre vient tuer le père ou le fils: consubstantialité du meurtrier et de sa victime. Il faut dire que cette anagramme lui est envoyée en réponse à un désespoir avoué, provenant de l'annonce publique de son exemption à participer à la guerre d'Algérie ${ }^{31}$.

25 février [1958] Je vais ce soir me saouler à mort. Ye souis désespéré. Mon exemption d'Algérie prononcée officiellement à midi. Je suis le bourreau. L'adjuvant qui la signifiait annonçait à voix haute devant toute la compagnie réunie (300 hommes):

En vertu de la loi du... l'exemption d'Algérie accordée à Georges

Perec, Père mort pour la France. Ça va loin? Du coup je deviens bon tireur. 32

On sent là comme une honte sinon une horreur à se faire ainsi désigner publiquement comme le fils de... ce père «mort pour la France». Horreur aussi de tout ce qui pourrait laisser entendre quelque chose comme «mon semblable, mon père». Le malheur est là, dans cet enfermement de l'histoire qui a fait de Georges Perec d'abord un orphelin, un survivant, une victime de la guerre, lui qui n'a été le témoin de rien, qui n'a été témoin que de ce rien. "Je suis le bourreau", écrit Perec, comme pour affirmer contre toute évidence l'envers de cette place soudain publiquement révélée, qu'il honnit. Dans l'un des fragments autobiographiques qui rappellent le départ de Perec en zone libre par le convoi de la Croix-Rouge, le fils semble porter ses titres comme une blessure:

La Croix-Rouge évacue les blessés. Je n'étais pas blessé. Il fallait pourtant m'évacuer. Donc, il fallait faire comme si j'étais blessé. C'est pour cela que j'avais le bras en écharpe. Mais ma tante est à peu près formelle: je n'avais pas le bras en écharpe, il n'y avait 
aucune raison pour que j'aie le bras en écharpe. C'est en tant que "fils de tué» "orphelin de guerre» que la Croix-Rouge [...] me convoyait. Peut-être, par contre, avais-je une hernie et portais-je un bandage herniaire, un suspensoir. (Wse: 80)

Le souvenir effectue à l'évidence un déplacement. La fausse blessure, qui est peut-être aussi un faux souvenir, vient manifestement recouvrir les noms que le sujet ne veut pas recevoir. Le nom du père lui-même sera frappé d'une certaine ambiguité, nié dans son origine juive et polonaise par l'adolescent qui fait mine de l'avoir toujours ignoré. «Il avait un nom sympathique: André. Mais ma déception fut vive le jour où j'appris qu'il s'appelait en réalité [...] Icek Judko, ce qui ne voulait pas dire grand chose» (Wse: 47). Si, dans la note qui se rattache à ce passage, Perec nous «traduit» le prénom en Isaac et Jehudi (sans oser davantage affirmer que ce mot veut justement dire «juif»), sa résistance à porter le fardeau de cet héritage le conduit à ne pas aller plus loin dans l'explication du nom. Un faux souvenir fera croire un moment au narrateur que ce père fut "dans les transmissions», ce qui est une manière de se signifier à soi-même ce que «veut dire» le nom du père. Mais c'est le nom d'André qui reviendra comme un pur souvenir de mot: «Croix de Saint-André», qui permettra à l'écrivain de décliner, à partir du X que représente cette croix, la série des lettres qui composent l'autobiographie (le double $\mathrm{V}$ que l'on retrouve dans le $\mathrm{X}$ et qui pointe les lieux - la rue Vilin, Villard-de-Lans - que le souvenir d'enfance convoque; les deux triangles qui forment l'étoile juive, la croix gammée qui s'obtient en prolongeant les branches du X, elle-même se composant de deux segments qui, séparés, donnent le sigle SS; le double X du Dictateur de Chaplin). Ce X rattaché au nom du père est «souvenir du mot, seul souvenir de cette lettre devenue mot", signe du mot "rayé nul, de l'ablation, de la multiplication, de la mise en ordre (axe des X) et de l'inconnu mathématique" (Wse: 109-110). Il est donc assez clair que ce père, inconnu, ce $\mathrm{X}$, est en même temps l'inépuisable géniteur de la lettre, du texte, du symbolique dont l'écrivain se fait le maître et l'ordonnateur.
C'est dans cette réversibilité du père et du fils, du bourreau et de la victime, du géniteur et de l'enfant, que se joue, me semble-t-il, le sens de l'œuvre de Perec; réversibilité douloureuse qui s'organise comme un travail, un "mode d'emploi» pour la vie, d'abord, mais aussi pour la préservation d'un noyau intouchable, insécable qui porte, quant à lui, tous les noms de la mère. Ce sont ces noms secrets qu'il faudra maintenant entendre dans $W$ ou le souvenir d'enfance, les noms que produit la dialectique toujours présente, voire obsédante, du jeu, celui d'un fort/da qui divise le sujet de la contrainte entre la douleur et la jouissance. Les lettres sont bien, chez Perec, des signifiants, des noms-du-père qui permettent d'aménager le champ catastrophique d'une disparition: celle de la mère. La question qui tenaille le sujet perequien ressemble à s'y méprendre à celle qui occupe les grands mystiques chrétiens: «Où est le corps?», demande Marie-Madeleine devant le tombeau vide. S'il y a bien, chez Perec, la recherche d'un corps glorieux à l'envers de celui absent au tombeau, ce corps re-suscité sera à la mesure d'une jouissance où l'endeuillement tourne à la férocité. Cette tonalité est, là encore, tributaire du rapport au père pour lequel l'enfant Perec dit avoir éprouvé un amour qui s'intégra à une "passion féroce" pour les soldats de plomb (Wse: 48).

\section{«ON LÂCHE UN ENFANT»}

\footnotetext{
Ma mère m'accompagna à la gare de Lyon. [...] Seize ans plus tard, en 1958, lorsque les hasards du service militaire ont fait de moi un éphémère parachutiste, je pus lire, dans la minute même du saut, un texte déchiffré de ce souvenir: je fus précipité dans le vide; tous les fils furent rompus; je tombai, seul et sans soutien. Le parachute s'ouvrit. La corolle se déploya, fragile et sûr suspens avant la chute maîtrisée. (Wse: 80-81)
}

La description de l'île W commence au chapitre XII qui ouvre la deuxième partie du livre. Si l'on en doutait encore, on se convainc, dès la première phrase, du statut hypothétique de ce lieu: «Il 
y aurait, là-bas, à l'autre bout du monde, une île. Elle s'appelle W» (Wse: 93). Le conditionnel n'échappe à personne et donne son registre subjectif à cette fiction de fantasme. Qu'est-ce qu'un fantasme? Freud le définissait comme un scénario imaginaire, conscient lorsqu'il prend la forme d'une rêverie, ou inconscient et déterminant, de là, les actes et les pensées du sujet. La plus intéressante fonction psychanalytique du fantasme est sans doute celle que Freud met en place dans son article intitulé «Un enfant est battu». Cet énoncé, «avoué avec une fréquence étonnante» par ses analysants, lui permet de penser l'origine du masochisme ${ }^{33}$.

D'après ce que nous savons actuellement, un tel fantasme, surgi dans la prime enfance peut-être dans des occasions fortuites et maintenu en vue de la satisfaction auto-érotique, ne peut être conçu que comme un trait primaire de perversion. [...] Nous savons qu'une telle perversion infantile peut ne pas persister pour la vie, qu'elle peut encore succomber plus tard au refoulement, avoir pour substitut une formation réactionnelle ou être transformée par une sublimation. ${ }^{34}$

Il semble y avoir quelque chose de cette «perversion» dans la fiction W, qui pourrait s'énoncer, suivant une légère variante: «Un enfant est lâché", avec toute la violence que ce terme implique dans l'ensemble du livre de Perec. Si le fait d'être lâché renvoie ici au souvenir d'avoir été "précipité dans le vide" par la séparation d'avec la mère, le terme garde aussi la résonance d'un abandon, sinon d'une trahison, dont on retrouve partout dans l'autobiographie les allusions teintées de reproches sourds mais chaque fois perceptibles. Être lâché signifie aussi, par endroits, être l'objet d'une négligence, comme si l'amour de la mère n'arrivait pas à protéger l'enfant de son inconscience à elle, de son ignorance que Perec n'hésite pas à inscrire dans ses élaborations fantasmatiques. Être lâché, c'est aussi ce qui désigne, au chapitre XXX de la fiction W, ce moment où les enfants, maintenus depuis leur naissance dans le gynécée, et pour ainsi dire préservés jusqu'à leur quinzième année des lois déchaînées qui sévissent sur l'île, sont brusquement lancés dans la vie de novice et d'athlète à laquelle ils sont depuis toujours destinés. Le cauchemar est d'autant plus terrifiant que rien dans l'enfance n'a préparé ce moment; au contraire, les conditions du bonheur et de l'innocence sont cultivées avec d'autant plus de cruauté qu'elles fondent la puissance traumatique de la révélation retardée.

Mais être lâché est en fait le principe générique de la Loi W qui n'accorde aucun «droit» durable et défait, à la première occasion, les filiations instaurées par la victoire, la force ou le décret:

Dès la fondation de $W$, il fut décidé que les noms des premiers vainqueurs [...] seraient donnés à tous ceux qui leur succéderaient au palmarès. [...] L'abandon des noms propres appartenait à la logique W: bientôt l'identité des Athlètes se confondit avec l'énoncé de leurs performances. (Wse: 133-134)

Cet enfant «lâché», autant dire "détaché», non lié, désarrimé de la filiation, ressemble à «[...] ces dessins dissociés, disloqués dont les éléments épars ne parvenaient presque jamais à se relier les uns aux autres et dont, à l'époque de W [du fantasme infantile], entre, disons, ma onzième et ma quinzième année, je couvris des cahiers entiers» (Wse: 97). Si je formule ainsi le «fantasme» qui se déploie dans tout le livre, c'est aussi parce qu'on peut lire, au centre de la description minutieuse et de plus en plus fascinée des pratiques de $\mathrm{W}$, quelque chose comme une scène originaire dans laquelle le motif du "lâcher » trouve toute sa portée mortelle et sadique (ce que le fantasme masochiste recouvre toujours). La chose est racontée au chapitre XXVI.

La conception des enfants est, sur W, l'occasion d'une grande fête que l'on appelle l'Atlantiade. [...] Les Atlantiades ont lieu à peu près tous les mois. On emmène alors sur le Stade central les femmes présumées fécondables, on les dépouille de leurs vêtements et on les lâche sur la piste où elles se mettent à courir $d u$ plus vite qu'elles peuvent. On leur laisse prendre un demi-tour d'avance, puis on lance à leur poursuite les meilleurs Athlètes W [...]. Un tour de piste suffit généralement aux coureurs pour rattraper les femmes, et c'est le plus souvent en face des tribunes d'honneur, soit sur la cendrée, soit sur la pelouse, qu'elles sont violées. (Wse: 169; je souligne) 
$\mathrm{Si}$, du côté des femmes, le terme de la course est le viol, du côté des hommes, le massacre et la mort sont à peu près assurés puisque, outre la sélection «sportive» longuement expliquée par le narrateur, qui a pour effet de favoriser les «coureurs de demi-fond ou, à la limite, les sprinters de $400 \mathrm{~m}$ » (Wse: 169), la course fait place à une «sélection naturelle» qui prend des proportions paroxystiques au fur et à mesure que la description, apparemment emportée par la vision qu'elle construit, se déploie. Le narrateur, manifestement admiratif de la logique de l'Administration W, s'attarde donc, pour commencer après nous avoir éclairés sur le but sexuel et procréateur de la course -, à décrire le parcours de ces géniteurs potentiels; seuls quelques-uns recevront, avec les honneurs de la victoire, le nom de Casanova dont la saveur ironique constitue la part la plus glorieuse de leur aveugle paternité. On apprendra au chapitre XXVIII que, à la différence des autres compétitions où les Athlètes sont placés sur la piste dans des cages qui s'ouvrent au moment du départ, on parque ensemble, lors des Atlantiades, les cent soixante-seize concurrents sur la zone de départ. Un treillis de fer électrifié, large de plusieurs mètres, est donc posé sur la piste, séparant les deux sexes; et ce n'est qu'après le demi-tour de piste effectué par les femmes que le courant sera coupé pour lancer les hommes «à la poursuite de leur proie» (Wse: 178). Au chapitre XXVI, le narrateur avait commencé sa description en nous disant que "pour compenser [les] différences et établir un tant soit peu l'équilibre", toutes les manœuvres qui visent la chute de l'adversaire sont permises (croche-pied, poussée, percussion, strangulation, morsure, énucléation, coups au sexe, etc.), sauf le port d'arme que l'on rend impossible par la nudité des coureurs, mais que l'on compense par le port de chaussures qui, nous assure le narrateur, se «justifie» pleinement puisqu'il s'agit « tout de même d'une course à pied "-, chaussures dont les pointes sont aiguisées et rendues particulièrement acérées et lacérantes. Le chapitre XXVIII se concentre donc sur l'«attrait exceptionnel» du spectacle que constituent ces Atlantiades. Le ton, en effet, atteint là une sorte d'acmé qui correspond tout à fait à l'escalade de violence qu'occasionne l'acte de copulation (par ailleurs inexistant en dehors de ces "compétitions»). Voici à quoi sont soumis les aspirants au titre de Casanova et seuls promus à la fonction d'engendrement. Le départ est donné:

Mais il ne s'agit pas, même au sens strict, d'un départ. En fait,

la compétition, c'est-à-dire la lutte, a commencé depuis

longtemps. Un bon tiers des concurrents sont déjà éliminés, les

uns parce qu'ils ont été assommés et qu'ils gisent inanimés sur le

sol, les autres parce que les coups qu'ils ont reçus [...] les rendent

inaptes à accomplir une course [...]. Sans doute cette lutte

permanente, dont la compétition elle-même n'est que le point

final, est-elle l'une des grandes Lois de la vie W [...]. Les autres

compétitions se déroulent dans un silence total. [...] Dans les

Atlantiades, au contraire, la foule peut, ou plutôt doit hurler tout son saoul et ses cris, captés, sont retransmis à pleine puissance par les haut-parleurs disposés tout autour du Stade. [...] Les vociférations et les clameurs sont telles [...], elles atteignent [...], lorsque les rescapés parviennent enfin à s'emparer de leurs proies pantelantes, un paroxysme tel que l'on pourrait presque croire à une émeute. (Wse: 178-181)

On trouve, dans ces pages, tous les éléments d'un théâtre très apparenté à une «scène originaire» qui ne saurait se raconter autrement que par la mise en scène d'une somme d'excitations (plaisir/déplaisir) excédant, par leur intensité et leur brutalité, les capacités du sujet à les élaborer psychiquement, c'est-àdire à leur donner sens dans le cadre de son histoire. Le «fantasme originaire» met en effet l'accent sur le mouvement, la formation, les traits déterminants de la représentation: castration, séduction, mode du rapport sexuel des parents suivant un scénario qui tente de retrouver, dans la surenchère, ce qui s'est perdu de la causalité. Il s'agit en fait d'une mise en ordre et en récit des données de l'expérience. On pourrait dire que, dans le fantasme $\mathrm{W}$, l'origine et les figures de la filiation se donnent sur le mode «ethnologique" de la description qui procède, par la voix off du narrateur-spectateur, à la symbolisation de l'origine de son histoire.

C'est en tout cas ce morceau particulièrement cruel, qui désigne l'origine par un viol, qui semble 
destiné à obturer le réel de l'angoisse que suscite, chez Perec, ce qui peut sans hésitation être désigné comme un viol de l'origine - la mère, disparue sans laisser de traces, entraîne avec elle toute possibilité d'une mémoire de son corps, de sa voix, de son regard. Dans la scène fantasmée, on repère de manière extrêmement prégnante le regard et la voix, parties du corps qui se prêtent particulièrement bien à l'opération logique de détachement qui transpose son objet dans l'imaginaire ${ }^{35}$. En tant qu'objets réels, ils sont irrémédiablement perdus, mais ils sont aussi le résultat d'une opération logique de détachement qui les restaure dans l'imaginaire: morceaux du corps propre auxquels nous n'avons pas accès, le regard et la voix sont, dans le fantasme perequien, élevés à la dignité optique du spectacle et à la puissance sonore du hurlement, donnant à la scène du viol son registre orgasmique pris en charge par le public en délire. Le regard et la voix, qui sont aussi, dans l'imaginaire, les morceaux perdus du corps de la mère, accèdent ici à une sorte d'ubiquité. La jouissance exacerbée semble en effet être la part de ceux qui - inconnus et impossibles à désigner précisément, à l'instar du narrateur, et qui le représentent à l'évidence occupent la place devenue collective de «témoin».

Comme dans tout fantasme, le sujet s'y représente de plusieurs manières. Tantôt il est ce coureur à cloche-pied, tantôt ce concurrent poussé, bousculé, transpercé qui trouve, dans les blessures, les traces tangibles de son histoire sans traces ${ }^{36}$. De même, les figures violentes de la castration, la guerre instaurée pour le triomphe du viol, la lâcheté promue au rang de nécessité travestissent la misère sexuelle et l'impuissance en Loi, selon un dispositif qui cherche à saturer toutes les pratiques corporelles d'agression, de manière à empêcher le surgissement d'un corps à corps autrement désiré.

Le fantasme perequien est entièrement fabriqué avec les mots du rapt et du viol, ce viol qui arrive dans son histoire comme arrachement de l'histoire, coup de hache qui morcelle non seulement le corps - en érotisant du coup chacun des morceaux, les phallicisant selon une jouissance dispersée et irrepérable -, mais aussi la mémoire qui se voit également frappée d'impuissance et qui s'accroche à des outils parcellaires (photos, documents, notes, etc.), sans pour autant parvenir à sa pleine existence. Cette fabrication a d'ailleurs pour fonction de faire oublier la cassure derrière l'écran du fantasme. L'agression est ainsi projetée sur l'ensemble des personnages, permettant à l'enfant d'être à la fois le bourreau et la victime; bourreau d'autant plus triomphant qu'il accomplit, avec $\mathrm{W}$, le tour de force de hausser sa jouissance au registre du témoignage, donnant à l'énonciation toute sa prégnance d'accomplissement du désir. La vengeance envers la mère se réalise ainsi dans cette scène originaire où c'est bien elle qui est «lâchée» puis violée, non pas tant par les «rescapés» qui sont autant de figures du Moi, que par l'écriture qui vient signer cette catastrophe. C'est l'impuissance de la mère à sauver son enfant (par son propre salut) qui trouve, dans W, les déformations les plus spectaculaires mais toujours reconnaissables. Et si les mères ne sont, dans cette histoire, que des femmes violées, c'est-à-dire par avance sans possibilité de faire valoir un désir d'enfant, c'est aussi pour transfigurer en Loi ce qui apparaît d'abord comme une accusation portée contre la mère et les femmes en général, reproche inséparable d'une culpabilité éprouvée par l'enfant lâché, abandonné. On retrouve là l'enfant survivant à l'œuvre, destituant non pas la mère mais la maternité en tant que telle, et tuant non pas le père mais le géniteur, potentiel ou victorieux, au nom d'une Loi (et d'une paternité) pour la mort.

\section{LES NOMS DE LA MÈRE}

Cyrla Schulevitz [13], ma mère, dont j'appris, les rares fois que j'entendis parler d'elle, qu'on l'appelait plus communément Cécile [...].

[13] J'ai fait trois fautes d'orthographe dans la seule transcription de ce nom: Szulewicz au lieu de Schulevitz. (Wse: 49, 59)

La description de W avance sans explications, suivant en cela l'exemple de Robert Antelme dans L'Espèce humaine ${ }^{37}$ dont Perec admirait l'écriture. Elle cherche 
assurément à construire, à inventer le témoignage impossible d'une disparition, d'un anéantissement. Si l'on a pu dire de l'écriture d'Antelme qu'elle « rompait absolument avec toute complicité sadomasochiste, toute ombre ambiguë de jouissance ${ }^{38}$, il faut dire de l'écriture de Perec qu'elle inscrit, pour sa part, les souffrances de l'espèce soumise à l'arbitraire de la Loi en se livrant à l'équivoque d'un enthousiasme de plus en plus appuyé dans l'horreur, qui n'a d'égal que le «rien»- l'absence de trace - sur lequel il se déchaîne. L'obsession des nombres, qui donne à la description de l'île sa rigueur dans le détail, son style halluciné, et qui fonde les lois de W sur une arithmétique imparable autant qu'arbitraire, se révèle être un interminable calcul effectué à partir des "nombres" de la mère - disparue officiellement le 11-2-43 - et du fils, né le 7-3-36 dans le 19e arrondissement ${ }^{39}$.

Le corps de la mère, absent au tombeau, est ainsi ressuscité - pour ne pas dire indéfiniment re-tué dans ce «jeu» catastrophique où le nombre vient produire, certes, le monument de sa disparition, mais aussi le corps brisé, rompu, «lâché» de l'enfantnarrateur survivant. Si W est une parabole des camps de la mort (encore que Perec ne semble pas se soucier de distinguer les camps d'extermination des camps de concentration et qu'il choisisse plutôt les témoignages de non-juifs pour modèles), on constate que la mère occupe dans ce récit une place matricielle éminemment mortifère. La voix, envoûtée finalement par la «raison» W, est donc bien celle du fils qui opère par la «musique» du nombre la saisie de cet effacement, saisie qui ne saurait se «représenter» autrement que dans la violence sadique et masochique la plus radicale.

À la lecture des chapitres consacrés à la description des lois $\mathrm{W}$, on comprend très tôt que ces lois relèvent d'une logique de l'arbitraire; bien plus: qu'elles constituent l'arbitraire en Loi. On sera dès lors frappé de lire, dans le récit autobiographie, que cet arbitraire se trouve accolé à la mère, qu'il en définit d'une certaine manière, pour l'enfant, la matière qui est à la fois son souvenir effacé et une part de son être. «L'image que j'ai d'elle, arbitraire et schématique, me convient; elle lui ressemble, elle la définit, pour moi, presque parfaitement» (Wse: 51). Cet arbitraire, qui s'associe tantôt à de la négligence, tantôt à de l'inconscience si ce n'est de l'irresponsabilité, scande le récit autobiographique comme une ponctuation sourde et insistante qui met à nu la part de ressentiment obscur et inassumable, voire insoutenable, qui mine le sujet "marqué» par cet arbitraire.

Le réseau des blessures et cicatrices que trament les souvenirs d'enfance compose à lui seul un texte où se donne à lire, en effet, la ronde des identifications perequiennes. C'est une maladresse de la mère qui inaugure la chaîne des blessures fictives et réelles qui traverse le récit d'enfance. Le souvenir flottant d'une blessure que la mère se serait accidentellement infligée, et rapporté dans le texte en caractères gras «écrit quinze ans plus tôt», est précédé d'une note, contemporaine de l'écriture du livre, qui taxe d'arbitraire l'énumération des maladies infantiles qui auraient "noirci» l'enfance. Cette note semble vouloir corriger cette mémoire hasardeuse par l'évocation de cicatrices encore visibles sur le corps du fils (implicitement données comme des preuves cette fois incontestables). Le déplacement de la blessure, qui passe des mains de la mère à celles du fils (ou l'inverse), donne d'emblée le principe d'apparition de toutes les autres blessures rapportées dans l'autobiographie (celle d'une petite fille dont la cuisse fut «transpercée», le souvenir du bras en écharpe, de l'omoplate fracturée, etc.): une assomption en miroir qui a pour effet de faire entrer la blessure (ou ses signes) dans un système d'échanges allant de l'autre à moi. Imposant sa fonction spéculaire, la blessure constitue, semble-t-il, l'un des traits les plus aptes à représenter la filiation maternelle.

Je naquis au mois de mars 1936. Ce furent peut-être trois années
d'un bonheur relatif que vinrent noircir sans doute les maladies de
ma prime enfance (coqueluche, rougeole, varicelle) ${ }^{[24]}$. La guerre
survint. Mon père s'engagea et mourut. Ma mère devint veuve de
guerre. Elle me mit en nourrice. Son salon fut fermé. Elle
s'engagea comme ouvrière dans une fabrique de réveille-matin. [...]
Il me semble me souvenir qu'elle se blessa un jour et eut la main
transpercée. Elle porta l'étoile.

Je naquis au mois de mars 1936. Ce furent peut-être trois années d'un bonheur relatif que vinrent noircir sans doute les maladies de ma prime enfance (coqueluche, rougeole, varicelle) ${ }^{[24]}$. La guerre survint. Mon père s'engagea et mourut. Ma mère devint veuve de guerre. Elle me mit en nourrice. Son salon fut fermé. Elle s'engagea comme ouvrière dans une fabrique de réveille-matin. [...] transpercée. Elle porta l'étoile. 
[24] Ces détails comme ceux qui précèdent sont donnés complètement au hasard. Par contre, je porte sur la plupart des doigts de mes deux mains, à la jonction des phalangettes, les marques d'un accident qui me serait arrivé alors que j'avais quelques mois: une bouillotte en terre, préparée par ma mère, se serait ouverte ou cassée, m'ébouillantant complètement les mains. (Wse: 52, 61)

Ouvertures et cassures, ces «motions" sont aussi celles du souvenir et de l'écriture; elles constituent les «lieux» où le sujet se reconnaît et où il peut, suivant une répétition constamment déplacée et pourtant indépassable, se refaire une filiation, se signifier à ce qui reste en souffrance, s'inventer une causalité. En bon auto-analyste, Perec reconnaît à ces blessures la fonction de substitution et la charge de jouissance qu'elles charrient:

[...] je vois bien ce que pouvaient remplacer ces fractures éminemment réparables [...] même si la métaphore, aujourd'hui, me semble inopérante pour décrire ce qui précisément avait été cassé et qu'il était sans doute vain d'espérer enfermer dans le simulacre d'un membre fantôme. (Wse: 113-114)

Le souvenir, cependant, continue de promener sur le corps la brisure, dans les hésitations qui lui font s'approprier la blessure, l'écharpe, le bandage de l'autre. Dans la valse tourbillonnante des identifications, ce n'est pas tant l'identité qui se définit que les modes de son inscription dont les miroirs constituent la surface de marquage.

[...] Plus simplement, ces thérapeutiques imaginaires, moins contraignantes et tutoriales, ces points de suspension, désignaient des douleurs nommables et venaient à point justifier des cajoleries dont les raisons réelles n'étaient données qu'à voix basse. (Wse: 114)

Perec parle à l'imparfait, mais c'est le souvenir, son actualité, plus que l'expérience réelle passée, qui ne cesse de mettre de l'avant ce corps imaginaire, ou cet imaginaire fait corps.

Les morceaux de corps empruntés au passage, ces membres fantômes, disent bien le statut spéculaire de la blessure, mais sa fonction oblitérante est déjà un travail symbolique dont le corps propre se fait l'écran de projection. La mère disparue sans laisser de trace fait retour dans la chair réelle, donnant au sujet la marque de son absence suivant une filiation au corps à corps dont seule la blessure témoigne. Les noms de la mère, on peut déjà le pressentir, ne libèrent pas le sujet de l'emprise du corporel et ne lancent pas les signifiants dans un travail de deuil d'où l'enfant pourrait «sortir» renommé. Ils inscrivent dans la chair une filiation secrète, indéchiffrable et pourtant «incarnée», consistante, qui interdit le deuil dans la mesure où c'est le corps propre qui se fait le réceptacle d'une trace devenant par là «signe». C'est ce qu'on peut lire dans le souvenir d'un autre accident, provoqué cette fois par Perec, aux sports d'hiver, pendant la guerre:

Un jour, un de mes skis m'échappa des mains et vint frôler le visage du garçon qui était en train de ranger ses skis à côté de moi et qui, ivre de fureur, prit un de ses bâtons de ski et m'en porta un coup au visage, pointe en avant, m'ouvrant la lèvre supérieure. [...] La cicatrice qui résulta de cette agression est encore aujourd'hui parfaitement marquée. Pour des raisons mal élucidées, cette cicatrice semble avoir eu pour moi une importance capitale: elle est devenue une marque personnelle, un signe distinctif [...]. (Wse: 145; je souligne)

Ce souvenir est précédé de nombreux détails, que Perec dit conserver dans une fraîcheur remarquable, détails concernant l'équipement de même que certaines techniques du skieur, parmi lesquelles celle qui consiste à remonter la pente, «skis écartés en $\mathrm{V}$ ». Il décrit par ailleurs longuement les différentes «attaches rostrales» où se retrouvent «le filin métallique» en forme de «fer de lance» et les lanières servant au laçage dont le protocole «[...] me faisait l'effet d'une cérémonie capitale (aussi capitale [...] que put m'apparaître, plus tard, le laçage de la ceinture dans Les Arènes sanglantes, de Blasco Ibanez [...]) multipliant autant les risques de fracture grave que les chances de performances exceptionnelles" (Wse: 145). On voit bien comment le texte de Perec articule le souvenir d'enfance aux tortures de W, mais surtout, on entend, dans la chaîne des associations 
apparemment "lâches", les signifiants de la mère qui sont blessure, violence, agression, cicatrice, ouverture, cassure, bref: atteintes violentes au corps. Cette atteinte qu'est la perte indiscernable de la mère, devenue construction «sanglante», est donc bien l'expérience dans laquelle le sujet se reconnaît.

Si la suite de ce passage amène l'identification au tableau d'Antonello de Messine, Le Condottiere, qui traverse l'œuvre de Perec, le rapport «mal élucidé» du jeune homme à cette cicatrice trouve une résonance dans le chapitre XX de W consacré à la transmission du nom, et qui précède le chapitre autobiographique qu'on vient de lire. Cette blessure réelle permet en fait une identification au père soldat, puisque Le Condottiere est le "portrait d'un homme» désigné comme un chef d'armes (le condottiere est un mercenaire combattant souvent pour des villes étrangères). Le père soldat - «quand je pense à lui, c'est toujours à un soldat que je pense» (Wse: 46) s'est quant à lui engagé et a combattu pour la France dans un régiment d'étrangers. La cicatrice semble permettre une transmission d'identité, mais, encore une fois, sur le mode imaginaire, imposant son inscription sur la surface corporelle. La chaîne des associations rejoint à l'évidence le chapitre XX, où l'on apprend qu'à W le nom est remplacé soit par un sobriquet formé à partir d'un signe du corps, soit par le nom du premier champion reconnu dans l'épreuve remportée. «Les novices n'ont pas de noms. On les reconnaît à ce qu'ils n'ont pas de W sur le dos de leur survêtement, mais un large triangle d'étoffe blanche cousu la pointe en bas» (Wse: 134).

Ce triangle rappelle le $\mathrm{V}$ des skis écartés pour la remontée de la pente. Ce V est bien sûr la lettre dominante de tout le livre et résume à elle seule, en passant par le X et le W qui la contiennent, toute l'histoire de Perec. Ce triangle cousu la pointe en bas sera de nouveau évoqué au chapitre XXXII, mais cette fois le narrateur affirme que la pointe est cousue vers le haut, comme s'il s'agissait de dessiner, "par lapsus", l'étoile juive sur les vêtements des novices.

On enlève aux jeunes leurs menottes, leurs fers et leurs boulets et on leur remet l'insigne de leur nouvelle fonction: un large triangle d'étoffe blanche qu'ils cousent, pointe en haut, sur le dos de leur survêtement. (Wse: 199)

Cette étoile innommée est un trait de la mère qui l'a portée. On voit bien, de là, que les noms de la mère traces d'une atteinte qui vire à l'attentat - engendrent dans le fantasme W la rature que constitue la transmission du nom.

Le chapitre XX raconte en effet l'édification d'un système onomastique "aussi subtil que rigoureux", selon lequel l'Athlète victorieux d'une performance reçoit le nom du premier vainqueur dans cette discipline, nom attribué et retiré au gré des victoires et des défaites suivant une précarité où l'identité n'a de sens que sportive et provisoire. Parce qu'ils sont "héréditaires», c'est-à-dire transmis au novice remplaçant l'Athlète qui laisse son équipe, ces noms deviennent, dès la troisième génération, «des repères atones, à peine plus humains que les matricules officiels» (Wse: 135). Dans cette complexe et laborieuse transmission des noms, c'est une opération d'anéantissement de l'identité qui s'effectue. Ces filiations folles - déterminées par des performances qui font de tout champion un champion de la cruauté (subie et infligée) - inscrivent dans la fiction ce qui, pour Perec, s'est perdu avec le corps de la mère.

W est bien le livre de la "défilialité» qui semble vouloir abolir non pas tant la filiation que son absence. Comme si, en inventant les lois de W, l'écriture s'appropriait la violence de l'effacement et fabriquait en son nom la scène de la disparition du corps et du nom. Dans cette tentative incestueuse, par laquelle la langue est «contrainte» de rendre le corps absent sur lequel elle s'élève, la mère et le fils sont voués à occuper les mêmes places d'abandonneur/ abandonné, de lâcheur/lâché. La culpabilité d'avoir survécu à la mère n'est pas séparable du ressentiment envers celle qui a laissé partir son enfant seul en zone libre et s'est faite embarquée dans une rafle.

Dans le dédale de $W$ ou le souvenir d'enfance, se dégage un impératif de cruauté qui semble viser à «meurtrir la mère». S’il y a bien chez Perec un travail de symbolisation par lequel s'effectue ce que l'on peut 
appeler tout bonnement le meurtre du père, l'opération semble toutefois frappée d'impuissance. À l'encontre du scénario imaginaire qui cherche, par lui, à réaliser l'inceste avec la mère, ce meurtre a au contraire pour effet de libérer l'enfant de l'emprise maternelle en lui permettant d'accéder aux signifiants de sa jouissance et aux noms de son devenir. «Tuer le père», chez Perec, met cependant à nu une absence cruelle, un impossible à nommer qui a pour conséquence de rendre le symbolique suspect, au point de forcer le sujet, en proie à la toute-violence du non-lieu, à le soumettre à sa maîtrise. Il se trouve donc métamorphosé en corps de contraintes, suivant le fantasme qui voudrait abolir son pouvoir obscur et sa Loi. Le symbolique, en effet, opère seul ses combinatoires et associations par quoi le sujet s'institue dans la parole comme nommé, c'est-à-dire lié à une histoire qu'il peut certes ignorer, nier, reconquérir, interpréter, mais qui l'arrime à une lignée de désirs dont il est aussi l'effet.

\section{LA FILIATION DU MESSIE}

$$
\begin{aligned}
& \text { Comment sait-on que Jésus était juif? } \\
& \text { - À } 33 \text { ans, il était toujours célibataire; } \\
& \text { - Il a repris les «affaires de son père»; } \\
& \text { - Il croyait que sa mère était vierge; } \\
& \text { - Sa mère le prenait pour le Messie. } \\
& \text { (Blague juive) } \\
& \text { Comment sait-on que Perec était juif? } \\
& \text { - Son nom avait une consonance bretonne; } \\
& \text { - Il avait été baptisé; } \\
& \text { - Son père s'appelait André, sa mère Cécile; } \\
& \text { - Il manifestait une indifférence notoire } \\
& \text { pour tout ce qui touche à la judéité; } \\
& \text { - Son psychanalyste était catholique. } \\
& \text { (Blague inconnue) }
\end{aligned}
$$

Le réseau des associations que Perec déploie à partir de son patronyme occupent une place importante dans l'œuvre. On se souviendra du nom de Cinoc dans La Vie mode d'emploi, dont le narrateur raconte les multiples métamorphoses dues aux passages des frontières qui transforment graphie et prononciation. Dans W ou le souvenir d'enfance, le narrateur Perec raconte à son tour, mais de manière assez incertaine, comment son nom a subi les transferts d'une langue à l'autre. Ce patronyme est surtout l'occasion d'une erreur étymologique qu'il n'est pas indifférent de rappeler.

Le nom de ma famille est Peretz. Il se trouve dans la Bible. En hébreu, cela veut dire "trou", en russe "poivre», en hongrois [...], c'est ainsi que l'on désigne ce que nous appelons «Bretzel» ("Bretzel» n'est [...] qu'un diminutif (Beretzele) de Beretz, et Beretz, comme Baruk ou Barek, est forgé sur la même racine que Peretz - en arabe, sinon en hébreu, B et $P$ sont une seule et même lettre). (Wse: 56)

On se demande comment le minutieux et obsessionnel Perec peut errer avec autant de fermeté lorsqu'il s'agit de présenter les «racines» littérales de son nom. On se souviendra qu'il se trompe aussi dans l'épellation du nom de sa mère. Il y a manifestement un trouble qui s'installe lorsqu'il s'agit d'inscrire les noms de la filiation. Ici, c'est l'étymologie qui fait l'objet d'une sorte d'improvisation conduisant à une erreur concernant les lettres sémitiques. Ni en hébreu ni en arabe $\mathrm{B}$ et $\mathrm{P}$ ne forment une seule lettre. Il est vrai que l'alphabet hébreu a plutôt tendance à susciter, chez cet écrivain, une mémoire fantasmatique. Le premier souvenir reconnu comme tel dans l'autobiographie est celui d'une lettre. Elle apparaît dans le rappel d'une scène de déchiffrement qui met en acte d'une manière intense la fonction du regard, partagée entre l'enfant qui «lit» la lettre et la désigne aux spectateurs, et cette collectivité incarnée par tous les membres de la famille rassemblés autour de l'enfant merveilleux. La lettre déchiffrée dans un journal yiddish (langue écrite avec l'alphabet hébreu) est dessinée par le narrateur et ne correspond à aucune lettre connue. Elle pourrait être, à la limite, un $\mathrm{G}$ inversé comme en un miroir, initiale du prénom de l'enfant, ou un "pé» hébreu initiale du nom de l'auteur; bref une lettre-nom à l'instar du W qui donne son nom à l'île et que l'on suppose provenir de l'initiale d'un nommé Wilson qui l'aurait fondée, ou encore de l'ethnie de ses habitants, reconnus comme 
des WASP (Wse: 94-95). La scène du déchiffrement de la lettre n'est d'ailleurs pas sans rejouer, en en inversant les valeurs affectives, la scène du viol: elle en serait en quelque sorte la réplique sur un autre plan. À l'envers du spectacle horrible que suscite, dans le Stade, la conception des enfants, les hurlements de l'émeute sont ici des voix douces qui s'extasient, la violence et la cruauté faisant place à la réconfortante protection que représente l'encerclement des corps. "L'enfant qui vient de naître» est l'objet de tous les regards, le Stade immense étant devenu, dans cette scène, pièce exiguë de l'arrière-boutique.

Le premier souvenir aurait pour cadre l'arrière-boutique de ma grand-mère. J'ai trois ans. Je suis assis au centre de la pièce, au milieu des journaux yiddish éparpillés. Le cercle de la famille m'entoure complètement: cette sensation d'encerclement [...] est protection chaleureuse, amour: toute la famille, la totalité, l'intégralité de la famille est là, réunie autour de l'enfant qui vient de naître (n'ai-je pourtant pas dit il y a un instant que j'avais trois ans?), comme un rempart infranchissable. Tout le monde s'extasie devant le fait que j'ai désigné une lettre hébraïque en l'identifiant [...]. (Wse: 26)

Cette scène originaire, écrite à l'envers de celle qui se donne à lire au centre du fantasme W, en reprend cependant le dispositif scopique. Ici, ce n'est pas le théâtre de la copulation qui se donne à voir comme causalité de la naissance, mais celui d'un savoir qui fonde la vocation d' «homme de lettres». Savoir oublié ou refoulé, manifestement, puisque la lettre que l'enfant aurait reconnue est à présent méconnaissable. On peut donc lire les deux scènes dans leurs relations mutuelles et entendre dans ce «souvenir» le désir de recouvrement - et de rédemption - que suscite (et que suscitera toujours) la lettre. À l'émeute de la foule, répond l'émerveillement de la famille, au monde sauvage et sans nom du struggle for life, répond l'univers pacifié de la lettre et de ses lois, et, à l'absence radicale de filiation, répond l'«intégralité» de la famille. Que cette scène familiale soit un fantasme originaire, la lettre fictive et les noms que Perec lui donne en témoignent suffisamment. Cette lettre improbable est tantôt appelée "gammeth", tantôt «gammel», le narrateur tâtonnant évidemment autour du nom "guimel», quatrième lettre de l'alphabet hébreu, qui ne ressemble en rien au dessin reproduit par l'auteur. Dans la note qui complète ce passage, le narrateur suppose enfin que la lettre qu'il a tracée pourrait bien être un «men" ou « $\mathrm{M}$ ». Il faut pourtant dire qu'aucune lettre hébraiqque ne porte ce nom, le $\mathrm{M}$ se disant en hébreu «mem».

Dans la série d'erreurs qui compose le commentaire de ce souvenir, Perec place tout de même les mots les plus aptes à signifier la filiation. Évidemment, «gamète» ne saurait mieux dire (gamos: mariage) la copulation que voile toute cette scène. Le dictionnaire nous apprend qu'il s'agit d'une «cellule reproductrice sexuée contenant la moitié des chromosomes des autres cellules de l'organisme et qui, s'unissant à une cellule reproductrice de sexe opposé, forme l'œuf d'où sortira le nouvel être vivant» "40. La gamel(le) renvoie, quant à elle, à la fonction nourricière et, sans doute, au souvenir de cette période où l'enfant était sous-alimenté, lui qui arriva deux ans plus tard «rachitique» à Villard-de-Lans en 1941. Quant au «men» ou «mène», il apparaît dans le fragment de phrase qui le suit immédiatement, et qui laisse entendre que cette lettre accomplirait justement l'alliance, devenue depuis irreprésentable, sauf sur le mode de la violence et du sadisme, entre le versant maternel et le versant paternel de la filiation. Perec écrit en effet:

[La lettre que j'ai tracée] pourrait, à la rigueur passer pour un «men» ou «M». Esther, ma tante [la sœur du père], m'a raconté récemment qu'en 1939 - j'avais alors trois ans - ma tante Fanny, la jeune soeur de ma mère m'amenait parfois de Belleville jusqu'à chez elle. (Wse: 27-28; je souligne)

Ce $\mathrm{M}$ dit aussi la charge d'amour que $\mathrm{W}$ retourne en haine: «m'haine».

Il est par ailleurs évident que le narrateur veut donner à cette scène la portée «messianique» d'une vocation. L'identification au Messie est presque toujours, chez Perec, une boutade, dont le plus amusant morceau est sans doute celui que l'on retrouve dans Je suis né: 
Je suis né le 25 décembre 0000. Mon père était, dit-on, ouvrier charpentier. Peu de temps après ma naissance, les gentils ne le furent pas et l'on dut se réfugier en Égypte. C'est ainsi que j'appris que j'étais juif et c'est dans ces conditions dramatiques qu'il faut voir l'origine de ma ferme intention de ne pas le rester. Vous connaissez la suite... 41

La boutade n'empêche pas le sérieux, elle permet de dire, comme la dénégation, ce qu'on ne saurait reconnaître pleinement et qui pourtant ne cesse d'exiger de se faire entendre. Et l'insistance avec laquelle le narrateur de l'autobiographie multiplie les allusions nous autorise sans doute à reconnaître dans W ou le souvenir d'enfance une fiction messianique dont il importe, évidemment, de donner le cadre spécifique. La scène du déchiffrement de la lettre, "par son thème, sa douceur, sa lumière», s'apparente donc pour le narrateur à un tableau réel ou inventé qui pourrait être de Rembrandt et qui s'intitulerait "Jésus en face des Docteurs"(Wse: 27). Mais, parce que le souvenir - le narrateur y tient - convoque un enfant "nouveau-né», une correction s'impose: «Le tableau auquel je me réfère, s'il existe, est beaucoup plus vraisemblablement une Présentation au Temple» (ibid.).

Si on revient aux associations qui entourent le patronyme, la fausse étymologie se révèle évidemment elle aussi signifiante. "Peretz» correspond effectivement à la prononciation polonaise du nom qui, dans cette langue, s'écrit «Perec». «Peretz», nous dit le narrateur, se trouve dans la Bible. Le personnage ne semble pas pour autant intéresser celui qui, pourtant, le reconnaît apparemment comme son "ancêtre» mythique, dans la mesure où il s'agit de l'ancêtre du Messie. Et l'erreur qui lui fait trouver une racine commune à «Peretz» et «Bretzel» le conduit à "Baruk», nom d'un prophète biblique, mais aussi mot hébreu qui veut dire "béni». Dans le dédale de ces associations, cette "bénédiction» est sans doute celle qui hante la scène de La Présentation au Temple qui est consécration à Dieu d'un nouveau-né. On voit bien comment Perec se refait une filiation à travers toutes ces erreurs savamment disséminées dans le texte. Son baptême à l'âge de sept ans, pendant l'été 1943, qui s'impose comme une précaution supplémentaire et pour lequel il lui est arrivé plus tard d'éprouver un violent dégoût, occupe dans l'autobiographie la fonction d'une restauration de la figure maternelle ${ }^{42}$.

Outre les nombreuses allusions à l'enfant-Messie par lesquelles la mémoire rassemble ses morceaux qui sont, dans $W$ ou le souvenir d'enfance, autant de représentants du Moi, le blason d'Otto Apfelstahl constitue sans doute le condensé le plus précis du sens que prend cette identification. Parmi les cinq symboles qui composent ce blason, trois demeurent obscursau regard du narrateur: l'un est un serpent dont les écailles auraient été des lauriers, ce qui dit bien comment, dans cette histoire, la "Gloire» et le "corps glorieux» sont intimement liés au mal, à la souffrance et à la ruse. Un autre symbole représenterait en même temps une main et une racine; alors que le troisième serait à la fois un nid, un brasier, une couronne d'épines, un buisson ardent, un cœur transpercé, autant de figures qui superposent les références au Christ et à Moïse, mais également à la mère qui eut elle aussi la main «transpercée». La main-racine vient quant à elle rattacher toutes les figures ensemble, comme elle rattache le fils et la mère. Les mains du nourrisson, ébouillantées par la maladresse de la mère, rejoignent dans l'infini de l'élaboration fantasmatique la main transpercée de la mère et celle que l'on retrouve dans le blason. Cette main-racine est aussi celle qui manque cruellement au souvenir de la séparation à la gare de Lyon, qui ne parvient pas à reconstituer de manière certaine les corps irrémédiablement arrachés l'un à l'autre. La mère ne tient pas l'enfant par la main; tout dans cette scène racontée trois fois est désarrimé, et aucune «image» de la mère ne sera retrouvée, sauf cette main, percée, et ces autres, cicatrisées, qui se touchent par association et se renomment par le blason qui ressemble à s'y méprendre à une figure de rêve.

Si Peretz est l'ancêtre du Messie, Perec, en faux marrane, s'amuse à multiplier les «souvenirs» qui l'identifient au Christ. Le baptême de Perec s'est accompagné d'une espèce de conversion enfantine qui 
n'est pas sans mettre en acte la figure de la Mère. Il semble en tout cas que tout ce qui concerne cette place christique actualise là encore les «noms de la mère». La judéité se révèle, pour Perec, un héritage pénible qui le désigne comme victime; la figure du Christ, qui se glisse dans l'œuvre sur le mode ambigu de l'allusion légère ou du message crypté, remplit dès lors une fonction que l'on pourrait bien reconnaître comme un antijudaïsme qui serait sa réponse à l'absence dans la filiation, au néant de la transmission, à la coupure et au rapt que la figure du Messie chrétien est bien propre à signifier lorsqu'on est juif. Parlant de ce qu'il est venu chercher par son enquête sur Ellis Island, Perec écrit:

Ce ne sont en rien des repères, des racines ou des traces,/ mais le contraire: quelque chose d'informe, à la limite du dicible,/ quelque chose que je peux nommer clôture, ou scission, ou coupure,/ et qui est pour moi très intimement et très confusément lié au fait même d'être juif/ je ne sais pas très précisément ce que c'est qu'être juif/ce que ça me fait d'être juif [...]/une certitude inquiète,/ derrière laquelle se profile une autre certitude, abstraite, insupportable:/ celle d'avoir été désigné comme juif,/ et parce que juif victime/43

Chaque fois qu'il approche de la source juive, la perspective se diffracte et les repères s'égarent, faisant place à une imagination qui frise bien souvent l'arbitraire dans la mesure où l'écrivain fait mine de transmettre des informations alors qu'il erre et parle à l'aveuglette. Ou bien il s'agit là d'un symptôme que l'on peut analyser avec Marcel Bénabou comme «un mouvement naturel de recul, une hésitation par rapport à un domaine avec lequel il n'entretient plus aucune familiarité», un "fléchissement de l'attention" et une «absence de curiosité» qui participent du refoulement entourant la judéité ressentie comme raturée, voire encombrante, sinon "oubliée" ${ }^{44}$; ou bien il s'agit d'une manœuvre de diversion qui, par l'erreur systématique, pointe l'impossible assomption d'une vérité inaccessible à l'origine, et permet de glisser les signifiants par lesquels le sujet se renomme écrivain, c'est-à-dire géniteur et père-mère de ses œuvres.
La judéité «négative» de Perec est associée pour lui à la mère. Dans la période qui correspond aux années du service militaire, Perec envoie à son ami Jacques Lederer, qui vient de faire la connaissance d'une jeune fille rescapée des camps, une lettre où l'humour noir sert à peine d'exorcisme à l'horreur et à l'angoisse:

J'aime pas les ex-déportées. Primo c'est youtre, ensuite c'est tatouée. Si on y ajoute quelques petites expériences, vivisectionnistes et stérilisatrices, beuah, beuah, je doute que tu apprécies. Mais après-tout, c'est peut-être ce que tu voulais suggérer par la sibylline phrase: "physiquement, démolie à jamais». Tu sembles engagé dans un putain de sac de nœuds. Demande-lui donc si elle a connu Cyrla Sczulewicz, femme Perec (voir pièce adjointe, envoyée à titre documentaire, pour satisfaire $\grave{a}$ un besoin pressant de plaisanteries morbides). [...] Il valait mieux se faire crever à Auschwitz qu'en revenir. [...] Remarque que je m'en fous, et que pour ma part, il me serait très agréable d'apprendre que tu as baisé ton numéro matricule (à propos, fais bien attention à tes pyjamas, choisis-les unis, les rayures sont très mal portées ces jours-ci). 45

L'histoire a en effet coupé les fils de la généalogie, imposant à l'enfant un incessant bricolage qui vise à renvoyer les coups au lieu même de cette causalité (maternelle). La damnation provient de la filiation, elle est ce qui déclenche les scénarios incessants de vengeance, de meurtre et de substitution. Et c'est le corps propre qui se fait le dépositaire d'une impuissance à reconnaître "où sont ses véritables ennemis" (Wse: 218); corps contre lequel il attentera «un peu " avant de faire un bout d'analyse avec JeanBertrand Pontalis qui a su décrire avec précision et raffinement la complexité du cas ${ }^{46}$. Malgré son écoute quasiment fusionnelle, on suppose facilement que Pontalis ne pouvait, de par sa culture catholique dont ses essais manifestent très clairement la trace, rappeler Perec à ses «racines» juives. Sans doute cet empêchement fut-il, consciemment ou non, déterminant dans le choix que fit Perec de son analyste. Quoi qu'il en soit, l'écrivain témoignera après-coup du statut de la «retrouvaille» que cette analyse a suscitée. 
Cela a eu lieu, cela avait eu lieu, cela aura lieu. On le savait déjà, on le sait. Simplement quelque chose s'est ouvert et s'ouvre: la bouche pour parler, le stylo pour écrire: quelque chose s'est déplacé, quelque chose se déplace et se trace, la ligne sinueuse de l'encre sur le papier, quelque chose de plein et de délié. 47

S'il y a bien une tonalité messianique qui traverse W ou le souvenir d'enfance, il faudrait la reconnaître non pas tant dans l'utopie négative qui est à sa manière une «rédemption» en cours, que dans la façon de reporter à l'infini l'avènement du "souvenir», de ce qu'il y aurait à retrouver. Comme le Messager de l'Empereur de Kafka, qui franchit la distance sans jamais parvenir à destination, et comme celui qui à l'autre bout, destinataire de ce message, le voit venir et l'attend, W ou le souvenir d'enfance construit quelque chose comme un franchissement qui est une attente, une suspension de l'avenir. Quelque chose vient vers nous, la mémoire, l'histoire, quelque chose a lieu qui est le dire, le désir, son devenir. En quatrième de couverture Perec écrit: «cette rupture, cette cassure qui suspend le récit autour d'on ne sait quelle attente». Il n'y aura pas d'autre rédemption que cette attente de ce qui n'est pas encore écrit.

\section{N O TES}

1. Lederer (1997: 277).

2. Ibid. L'expression est de Perec et désigne, juste avant le passage précédemment cité, le statut qu'il entend donner à son œuvre encore à venir dont le premier roman est provisoirement intitulé: La Nuit.

3. Toutes les citations de W ou le souvenir d'enfance (Wse) proviennent de l'édition de 1993. Ce texte fut d'abord publié chez Denoël en 1975. 4. Voir P. Lejeune (1991).

5. Voir, entre autres, C. Burgelin, en particulier la troisième partie: "Les avatars du "cas" Perec" (1996: 85-113).

6. Entre autres, M. Bénabou, «Perec et la judéité», Cahiers Georges Perec, no 1 (1985: 15-30); R. Robin (1993).

7. Perec se situe explicitement, à la fin de W, sous le patronage de Robert Antelme et de David Rousset. Voir aussi A. Roche (1997) et A. Chauvin (1997).

8. La mort du père, Icek Judko Perec, survient le 16 juin 1940. La mort de la mère, Cyrla Szulewicz, demeure quant à elle impossible à retracer. Le train dans lequel elle fut embarquée avec des milliers d'autres, et qui devait la mener à Auschwitz, a quitté Drancy le 11 février 1943. Cette dernière recension de son existence sera la seule notation qui permettra aux autorités françaises d'indiquer les lieu et date officiels - faux lieu et fausse date - de sa mort.

9. S. Freud (1985a:269-287).

10. G. Perec (1990: 83).

11. Voir B. Magné, «Les sutures dans WSE», Cahiers Georges Perec, n² (1988).

12. «De ma mère, le seul souvenir qui me reste est celui du jour où elle m'accompagna à la gare de Lyon d'où, avec un convoi de la Croix-

Rouge, je partis pour Villard-de-Lans: bien que je n'aie rien de cassé, je porte le bras en écharpe. Ma mère m'achète un Charlot intitulé Charlot parachutiste: sur la couverture illustrée, les suspentes du parachute ne sont rien d'autre que les bretelles du pantalon de Charlot» (Wse: 45); «Un jour elle m'accompagna à la gare. C'était en 1942. C'était à la gare de Lyon. Elle m'acheta un illustré qui devait être un Charlot. Je l'aperçois, il me semble, agitant un mouchoir blanc sur le quai cependant que le train se mettait en route. J'allais à Villard-de-Lans, avec la Croix-Rouge" (ibid. : 52). «Ma mère m'accompagna à la gare de Lyon. J'avais six ans. Elle me confia à un convoi de la Croix-Rouge qui partait pour Grenoble, en zone libre. Elle m'acheta un illustré, un Charlot, sur la couverture duquel on voyait Charlot, sa canne, son chapeau, ses chaussures, sa petite moustache, sauter en parachute. Le parachute est accroché à Charlot par les bretelles de son pantalon " (Wse: 80). Au sujet des "erreurs" que fait Perec sur la date de ce départ qui eut lieu plutôt en 1941, et sur l'impossible existence à Paris à cette date d'un illustré représentant Charlot, voir P. Lejeune (1991: 82-85) et D. Bellos (1994: 85).

13. Dans L'Interprétation du rêve (chap. V, "Matériel et sources du rêve »), Freud écrit: ("[...] so dass man zu seiner Überraschung im Traum das Kind mit seinen Impulsen weiterleben findet»), «[...] si bien qu'on a la surprise de trouver dans le rêve l'enfant encore vivant (weiterleben: qui continue de vivre ou survivant) avec ses impulsions» (2003: 222).

14. Voir P. Lejeune (1991: 89).

15. Voir M. van Montfrans (1999: 155).

16. Je reviendrai plus loin sur ce souvenir.

17. G. Perec (1979: 3-4).

18. Tiré de l'exergue que Perec prévoyait pour L'Arbre. Histoire d'Esther et 
de sa famille qui aurait été «la description, la plus précise possible, de l'arbre généalogique de mes familles paternelle, maternelle et adoptive(s)" (ce livre ne fut pas écrit). Lettre à M. Nadeau, 7 juillet 1969, publiée dans Je suis né (1990: 53-54).

19. Devant la tombe de son père, Perec raconte avoir éprouvé «quelque chose comme une sérénité secrète liée à l'ancrage dans l'espace, à

l'encrage sur la croix, de cette mort qui cessait d'être ainsi abstraite [...]» (Wse: 59).

20. Sur l'intertextualité dans $W$ ou le souvenir d'enfance, voir $\mathrm{G}$.

Mouillaud-Fraisse, "Cherchez Angus. W: une réécriture multiple», Cahiers Georges Perec, n² (1988: 85-96).

21. M. Schneider (1985: 233-234).

22. On en trouve une mention explicite au chapitre XXVI: «La coutume veut en effet que l'on laisse vivre la totalité des enfants mâles (sauf s'ils présentent à la naissance quelque malformation les rendant inaptes à la compétition [...]), mais que l'on ne garde qu'une fille sur cinq"

(Wse: 168).

23. L. Lederer (1997: 278).

24. P. Lejeune (1991: 65).

25.S. Freud (1981: 52-53).

26. G. Perec (1969: 133).

27. S. Freud (1981: 54).

28. J. Lederer (1997: 278).

29. Ibid.: 115 (lettre du 2 mars 1957).

30. Ibid.: 121.

31. «Perec avait demandé à être dispensé d'Algérie au moment des trois jours et il savait que sa requête serait acceptée parce que son père était "mort pour la France" "(ibid.: 205).

32. Ibid.: 278.

33. S. Freud (1985b: 219-243).

34. Ibid.: 221.

35. J. Lacan (1966-1967: Séminaire XIV - inédit).

36. On trouve en effet, dans les fragments autobiographiques qui alternent avec la fiction, les souvenirs de sensations cinesthésiques de saut et de chute qui permettent de reconnaitre, dans les luttes entre les Athlètes de W, des mouvements qui sont en fait des représentants du sujet du fantasme.

37. G. Perec, «Robert Antelme ou la vérité de la littérature [1963]», dans R. Antelme (1996: 178-180).

38. C. Rabant, «Se soulever contre ce qui est là...», dans R. Antelme (1996: 121).

39. M. van Montfrans (1999: 222-223), c'est l'auteur qui souligne.

40. Dictionnaire Le Petit Robert.

41. Perec (1990: 10).

42. «Vers la fin des années 60 Perec donna l'impression, à certains de ses amis, de nourrir quelque amertume à l'endroit de cette conversion enfantine, accusant ses protecteurs d'avoir profité de la situation créée par la guerre pour "lui flanquer un baptême” ". D. Bellos (1994: 92). 43. G. Perec et R. Bober (1980: 40-41).

44. M. Bénabou, "Perec et la judéité», Cahiers Georges Perec, nº 1 (1985: 18-20).

45. Lettre du 12 mai 1958 (Lederer, 1997: 247-248).

46. Je fais allusion dans cette phrase à la tentative de suicide qui décida Perec à faire une analyse. Voir J.-B. Pontalis, «Sur la douleur

(psychique)» (1977: 263).

47. «Les lieux d'une ruse" (Perec, 1985: 61-62).

\section{RÉfÉREN CES Biblio g RAPHiQ U ES}

Cahiers Georges Perec, no 1 [1985]: Colloque de Cerisy (juillet 1984), Paris, P.O.L.

Cahiers Georges Perec, n 2 [1988]: Textuel 34/44, n²1, Paris, Université Paris VII.

ANTELME, R. [1996]: Textes inédits sur L’Espèce humaine. Essais et témoignages, Paris, Gallimard.

Bellos, D. [1994]: Georges Perec, une vie dans les mots, Paris, Seuil. Burgelin, C. [1996]: Les Parties de dominos chez Monsieur Lefèvre. Perec avec Freud, Perec contre Freud, Paris, Circé.

Chauvin, A. [1997]: Leçon littéraire sur W ou le souvenir d'enfance de Georges Perec, Paris, PUF.

FREUD, S. [2003]: L'Interprétation du rêve, Euures complètes IV, Paris, PUF;

__ [1985a]: "Construction dans l'analyse [1937] ", Résultats, idées, problèmes, Paris, PUF;

[1985b]: «Un enfant est battu », Névrose, psychose et perversion,

Paris, PUF;

[1981]: «Au-delà du principe de plaisir [1920]», Essais de psychanalyse, Paris, Payot.

LACAN, J. [1966-1967]: Séminaire XIV: La Logique du fantasme (inédit).

On peut toutefois se reporter au site Web de la Bibliothèque Lacan au [www.ecole-lacanienne.net/seminaireXIV.php].

LEDERER, J. [1997]: "Cher, très cher, admirable et charmant ami...", Correspondance G. Perec et J. Lederer (1956-1961), Paris, Flammarion. Lejeune, P. [1991]: La Mémoire et l'Oblique. Georges Perec autobiographe, Paris, P.O.L.

MonTFrans, M. van [1999]: Georges Perec. La Contrainte du réel, Amsterdam-Atlanta, Rodopi, GA.

Perec, G. [1993]: W ou le souvenir d'enfance, Paris, Gallimard, coll. "L'Imaginaire";

[1990]: Je suis né, Paris, Seuil, coll. «La Librairie du XXe siècle»;

[1985]: Penser/Classer, Paris, Hachette;

[1979]: Mots croisés, Paris, Mazarine;

_ [1978]: La Vie mode d'emploi, Paris, Hachette;

_ [1969]: La Disparition, Paris, Denoël.

PEREC, G. et R. BOBER [1980]: Récits d'Ellis Island. Histoires d'errances et d'espoirs, Paris, Éd. du Sorbier/INA.

PONTALIS, J.-B. [1977]: Entre le rêve et la douleur, Paris, Gallimard, coll. «Tel».

RoBIN, R. [1993]: Le Deuil de l'origine: une langue en trop, la langue en moins, Saint-Denis, Presses de l'Université de Vincennes.

Roche, A. [1997]: W ou le souvenir d'enfance de Georges Perec, Paris, Gallimard, coll. «Folio».

SCHNEIDER, M. [1985] : «Père ne vois-tu pas». Le père, le maître, le spectre dans L'Interprétation des rêves, Paris, Denoël. 